\title{
Comparison of perturbative and non-perturbative methods in $f(R)$ gravity
}

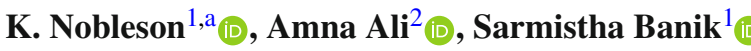 \\ ${ }^{1}$ Department of Physics, BITS Pilani, Hyderabad Campus, Jawaharnagar, Hyderabad 500078, India \\ ${ }^{2}$ Department of Mathematics, Jadavpur University, Kolkata, West Bengal 700032, India
}

Received: 20 August 2021 / Accepted: 22 December 2021 / Published online: 13 January 2022

(C) The Author(s) 2022

\begin{abstract}
In this work, we investigate the structure and properties of neutron stars in $R^{2}$ gravity using two approaches, viz: the perturbative and non-perturbative methods. For this purpose, we consider NS with several nucleonic, as well as strange EoS generated in the framework of relativistic mean field models. The strange particles in the core of NS are in the form of $\Lambda$ hyperons and quarks, in addition to the nucleons and leptons. In both the approaches, we obtain mass-radius relation for a wide range of values of the extra degree of freedom parameter $a$ arising due to modification of gravity at large scales. The mass-radius relation of the chosen equation of states lies well within the observational limit in the case of GR. We identify the changes in the property of neutron star in the background of $f(R)$ gravity, and compare the results in both the methods. We also identify the best suited method to study the modified gravity using the astrophysical observations.
\end{abstract}

\section{Introduction}

Einstein's General theory of Relativity (GR) has drastically changed our understanding of space and time [1]. To this date, it stands on a strong footing as it passed every single observational test we have ever thrown at it. Besides, impressive number of phenomena that general relativity predicts - which includes the presence of black hole, time dilation, gravitational redshift, gravitational lensing, gravitational waves have all been borne out of this century old classical theory of gravity. Yet general relativity is not the ultimate theory of gravity as it fails to explain primarily two main phenomena of Universe. First, it fails to explain the present cosmic acceleration without introducing 'Dark Energy', a gravity defying mysterious component, which comprises $70 \%$ of the Universe [2-5]. Second, it fails to explain the anomalous rota-

\footnotetext{
a e-mail: nobleson.phy@gmail.com (corresponding author)
}

tion curves of galaxies, the formation of large-scale structure via gravitational instability. Within the framework of GR, the solutions of these two problems demand existence of large amount of invisible mysterious energy (dark energy) and matter (dark matter), respectively. Faced with these theoretical issues the next natural consequence is to look for the modification of the Einstein' gravity at the large scale. Essentially, it assumes gravity acts in novel ways in the largest and smallest length scales compared to the well-tested middle length scales. So far, GR has been only tested with high accuracy within our own solar system. In addition, we know that, gravity is modified at a short distance [6] and hence there is no guarantee that it would not suffer any breakdown at large scales where it is never directly verified. Large-scale modifications of gravity could arise in variety of ways - for example, it might arise due to the extra dimensional effects or can be inspired by fundamental theories. Alternatively, they could also be motivated by phenomenological considerations such as $f(R)$ theories of gravity, where Einstein-Hilbert action that describes GR is modified which in turn leads to the scalar tensor theories [7]. Within these frameworks, the additional scalar degree of freedom can be tuned to mimic any type of viable cosmological evolution at any cosmological scales. The simplest of these modifications are $f(R)$ theories, where $f(R)$ has the higher order extensions of the Ricci scalar [8].

Though these modifications are well motivated and consistent, the overabundance of ideas and possibilities has led to the lack of one compelling theory which could actually describe gravity. To be a viable model of gravity, any largescale modification of gravity must reconcile with the local physics constraints by undergoing the solar system tests and laboratory tests. Majority of these models have undergone the solar system tests where the gravitational field is substantially weak and has been subject to numerous experimental tests, which confirms the accuracy of GR on the weak gravitational background. However, any consistent theory of gravity, classical or modified, should also be equally applicable 
to the strong gravity regime. The compact objects, such as 'Neutron stars' (NS) provide a good platform to study the behaviour of strong gravity. They provide a powerful probe of the GR in the strong-field regime and opens new windows to explore and constrain the modified theories of gravity.

To study NS in $f(R)$ theory of gravity, one can derive a set of modified Tolman-Oppenheimer-Volkoff (TOV) equations that describe a static, spherically symmetric mass distribution under hydrostatic equilibrium. Due to the presence of higher order curvature terms in the action, it is not trivial to derive the TOV equations. There are two methods proposed to tackle this problem: (a) perturbative approach [9-11,31-33] and (b) non-perturbative approach $[12,13]$.

In the perturbative approach, the modifications to the theory are considered as higher-order corrections to the GR. The effects of these corrections can be controlled by the free parameter and can be tuned to obtain the required correlation with observation. However, the interior and exterior solutions are not obtained by solving the equation simultaneously. A Schwarzschild solution is considered as the vacuum solution. In some papers, negative values of $a$ were considered which leads to tachyonic instabilities. In the nonperturbative approach, the interior and exterior equations are solved simultaneously and self-consistently to derive the TOV equations. Negative values of $a$ are not considered. In this paper, we use the above both approaches and examine the properties of NS and compare them. We will also comment on the impact of the free parameter in both approaches.

The highly degenerate interior of NS is described by the equation of state (EoS), that relates between a set of thermodynamic variables such as pressure, energy density, density, temperature, etc. At present there is no single EoS that an accurately model the NS core. Scientists do constrain the EoS by the nuclear physics experiments, such as the binding energy, compressibility, effective mass of nucleons, symmetry energy, and its slope. These observations are limited to normal nuclear matter density whereas the mass and radius data of the observed NS definitely conforms to a dense core that surpasses normal nuclear matter density ( $>\rho_{0}$ ). Constraints on NS masses and radii now come from a variety of astrophysical observations including pulsar timing, thermal and bursting X-ray sources, and the multimessenger gravitational waves events, specially GW170817, the first observed binary neutron star merger. In addition to the assumptions inherent to general relativity and causality of the EoS, the pulsar timing measurements of the most massive pulsars can limit the NS maximum mass from the lower end while the multimessenger GW events can convincingly set an upper limit and also constrain radius through tidal deformibility $(\Lambda)$ estimations $[14,15]$. The measurements of pulsar masses for PSR J1614-2230 (1.97 $\left.\pm 0.04 M_{\odot}\right)$ in 2010 and PSR J0348+0432 (2.01 $\left.\pm 0.04 M_{\odot}\right)$ in 2011 have tremendous implications for constraining the NS EoS. One of the most intriguing question one may ask is, if there exist strange degrees of freedom (hyperons, anti-kaon condensates, quarks) at the high-density core of the NS and whether their presence can change the waveform coming from the merger. Pauli exclusion principle strongly dictates the presence of strange hadrons in the high density baryonic matter. Regardless of precise compositions, any additional degrees of freedom results in a softer EoS, lowering the maximum mass predicted by EoS that is constructed for neutron, protons, and leptons only. The mass-radius data of PSR J0030+0451 published and recently updated by NASA's Neutron star Interior Composition Explorer (NICER) [16]. Researcher have estimated its mass and radius around $1.3-1.4 M_{\odot}$ and radius $\sim 12.7-13$ kilometers $[16,17] . \mathrm{M}=1.44_{-0.14}^{+0.15} M_{\odot}$, $\mathrm{R}=13.02_{-1.06}^{+1.24} \mathrm{~km} \mathrm{[17]}$ and $\mathrm{M}=1.34_{-0.16}^{+0.15} M_{\odot}$,

$\mathrm{R}=12.71_{-1.19}^{+1.14} \mathrm{~km}[16]$, to the $68.3 \%$ credibility interval, provide an additional useful constraint on the NS EoS.In this paper, we use the relativistic EoS which are compatible with the recent mass-radius observations. Also, we consider strangeness in the form of $\Lambda$-hyperons and quarks in addition to the nuclear matter.

The paper is organised as follows. In section II, we review the field equations of $f(R)$ modified gravity model considering its perturbative and non-perturbative forms. We obtain the modified TOV equations in both forms. In section III, we present an overview of the EoSs used in this work. In section IV, the modified TOV equations are solved numerically for various EoSs, the two different forms of $f(R)$, and various values of the free parameter $a$. Finally, in the discussion section, we comment on the results of numerical study and on the significance of the scale of the free parameter $a$ for $R^{2}$-modified gravity model, $f(R)=R+a R^{2}$.

\section{$2 f(R)$ gravity and the modified TOV equations}

NS are relativistic objects and their structure calculations are carried out in a GR framework. The interior of a static star is considered as a perfect fluid and its Schwarzschild metric is represented by the line element:

$d s^{2}=-e^{2 \phi(r)} d t^{2}+e^{2 \lambda(r)} d r^{2}+r^{2}\left(d \theta^{2}+\sin ^{2} \theta d \vartheta^{2}\right)$.

Here $\mathrm{m}(\mathrm{r})$ is the gravitational mass inside radius $r$ of a circle about the origin. In hydrostatic equilibrium, the structure of a spherically symmetric, static relativistic NS is determined by the Tolman-Oppenheimer-Volkoff (TOV) equations. These ordinary differential equations are:

$$
\begin{aligned}
& \frac{d m}{d r}=4 \pi \rho r^{2} \\
& \frac{d p}{d r}=-(\rho+p) \frac{d \phi}{d r} .
\end{aligned}
$$


where $\rho$ is energy density, $\mathrm{p}$ is pressure, both in the fluid frame. Given a barotropic EoS, i.e., the EoS can be written as $\rho=\rho\left(n_{B}\right)$ and $p=p\left(n_{B}\right)$, where $n_{B}$ is the baryon number density in the fluid frame the stellar structure can be computed by numerically integrating Eq. (2) from the centre at $\mathrm{r}=0$ out to the star surface at $\mathrm{r}=\mathrm{R}$. The boundary conditions are $p(r=R)=0, p(r=0)=p_{c}\left(\rho_{c}\right), m(r=0)=$ 0 . $\mathrm{M}$ is the enclosed gravitational mass given by $M(r)=$ $4 \pi \int_{0}^{R} \rho(r) r^{2} d r$.

In the modified gravity basically two approaches are followed: (A) Perturbative. (B) Non-perturbative.

\subsection{Perturbative method}

In the framework of perturbative approach, $f(R)$ is expressed as $f(R)=R+a h(R)+\mathcal{O}\left(a^{2}\right)$, where $h(R)$ is an arbitrary function of $R, a$ is the free parameter for perturbation, and $\mathcal{O}\left(a^{2}\right)$ denotes the possible higher-order corrections to $a$. In this work, we have considered $h(R)=R^{2}$, R being the Ricci curvature scalar. The quantities from the EoS, i.e., pressure and energy density are represented as zeroth order $p^{(0)}$ and $\rho^{(0)}$, the corresponding calculated mass in GR is $m^{(0)}$. The Ricci curvature scalar has to be evaluated at $\mathcal{O}(1)$ order, i.e.,

$R=R^{(0)}=8 \pi\left(\rho^{(0)}-3 p^{(0)}\right)$.

The modified TOV equations in the perturbative method are as follows:

$$
\begin{aligned}
\frac{d m}{d r}=4 \pi \rho r^{2}-a r^{2}\left[8 \pi \rho^{(0)} R-\frac{R^{2}}{4}\right] & \\
+a & {\left[\left(2 r-3 m^{(0)}-4 \pi \rho^{(0)} r^{3}\right) \frac{d R}{d r}\right] } \\
+a & {\left[r\left(r-2 m^{(0)}\right) \frac{d^{2} R}{d r^{2}}\right] } \\
\frac{r-2 m}{\rho+p} \frac{d p}{d r}= & 4 \pi r^{2} p+\frac{m}{r} \\
& -a r^{2}\left[8 \pi p^{(0)} R-\frac{R^{2}}{4}\right] \\
& -2 a\left(r-3 m^{(0)}+2 \pi p^{(0)} r^{3}\right) \frac{d R}{d r}
\end{aligned}
$$

The full derivation of this formalism can be found in [10, 11,31-33].

As in GR, the modified TOV Eqs. (4), and (5), can be solved numerically to obtain mass and radius of the NS.

We note that perturbation expansion parameter $a$ introduces a new scale into the theory. Further, by choosing a realistic EoS, we compute mass-radius relation for various values of $a$, thereby placing a bound on $a$ for perturbative $f(R)$ gravity model: $f(R)=R+a R^{2}$.

\subsection{Non-perturbative method}

In the non-perturbative method, we introduce a new field $\Phi=f^{\prime}(\psi)$ such that the scalar field $\varphi=\frac{\sqrt{3}}{2} \ln \Phi$. We define $A^{2}(\varphi)=\Phi^{-1}(\varphi)=\exp (-2 \varphi / \sqrt{3})$ and $\alpha(\varphi)=\frac{d \ln A(\varphi)}{d \varphi}=$ $-\frac{1}{\sqrt{3}}$. The full derivation of this formalism can be found in $[12,13]$. The modified TOV equations in the non-perturbative method are as follows:

$$
\begin{aligned}
\frac{d \lambda}{d r}= & e^{2 \lambda}\left[4 \pi \rho r A^{4}+\frac{r e^{-2 \lambda}}{2}\left(\frac{d \varphi}{d r}\right)^{2}\right. \\
& \left.+\frac{r\left(1-A^{2}\right)^{2}}{16 a}-\frac{\left(1-e^{-2 \lambda}\right)}{2 r}\right]
\end{aligned}
$$

$$
\begin{aligned}
\frac{d \phi}{d r}= & e^{2 \lambda}\left[4 \pi p r A^{4}+\frac{r e^{-2 \lambda}}{2}\left(\frac{d \varphi}{d r}\right)^{2}\right. \\
& \left.-\frac{r\left(1-A^{2}\right)^{2}}{16 a}+\frac{\left(1-e^{-2 \lambda}\right)}{2 r}\right]
\end{aligned}
$$

$$
\begin{aligned}
\frac{d^{2} \varphi}{d r^{2}}= & e^{2 \lambda}\left[\frac{A^{2}\left(1-A^{2}\right)}{4 \sqrt{3} a}-\frac{4 \pi A^{4}(\rho-3 p)}{\sqrt{3}}\right] \\
& -\frac{d \varphi}{d r}\left(\frac{d \phi}{d r}-\frac{d \lambda}{d r}+\frac{2}{r}\right)
\end{aligned}
$$

$\frac{d p}{d r}=-(p+\rho)\left[\frac{d \phi}{d r}-\frac{1}{\sqrt{3}}\left(\frac{d \varphi}{d r}\right)\right]$

where $\phi$ and $\lambda$ terms are taken from Eq. (1). In order to solve the above systems of differential equations for the interior and the exterior of the NS, we need EoS for the NS matter as well as impose appropriate boundary conditions. The dimensions of the parameter $a$ is in terms of $r_{g}^{2}$, where $r_{g}=1.47664 \mathrm{~km}$ corresponds to one solar mass.

\section{Neutron star EoS}

We describe the interior of a static NS, that is supported against the gravity by degeneracy pressure of the constituent fermions. The DD2 [18,19] and FSU [20] models are employed to represent the hadronic matter. The Fermi energy of the constituent fermions are quite high compared to the interior temperature of the NS, a zero temperature EoS is sufficient to represent the dense matter relevant to NS interior. Both the EoS are constructed following the original Walecka model [21] where the basic relativistic Lagrangian has the effective interaction through contribution from $\sigma, \omega$ and $\rho$ mesons without any self-coupling terms. The equation of 
motions are solved within the widely used relativistic meanfield (RMF) approach. The meson-baryon couplings of the DD2 EoS are density-dependent, they take care of the high density behaviour of the supra-nuclear dense matter. Also, the thermodynamical consistency is maintained through the rearrangement terms which arise due to density dependent couplings. However, the model parameters are fitted to the properties of finite nuclei at normal nuclear matter density $\left(n_{0}=0.149 \mathrm{fm}^{-3}\right)$ [22]. Also, the experimental mass values for the nuclei are used. DD2 has reasonable nuclear matter parameters: the binding energy per nucleon $a_{v}=16.02$ $\mathrm{MeV}$, incompressibility $\mathrm{K}=242.7 \mathrm{MeV}$, symmetry energy $31.67 \mathrm{MeV}$ and its slope coefficient 55.03 MeV [19]. The sub-saturation density part of the hadronic EOS is calculated with the statistical model with excluded volume and interactions of Hempel and Schaffner-Bielich [18]. The parameters of FSUGarnet, on the other hand are determined by fitting model predictions to experimental data, based on genuine physical observables that can be either measured in the laboratory or extracted from observation [20]. The EoS is stiff, the resulting values for the symmetry energy and its slope at saturation density are $30.92 \pm 0.47$ and $51.0 \pm 1.5 \mathrm{MeV}$ respectively, and are well within the acceptable range. The other parameters are $\mathrm{K}=229.5 \mathrm{MeV}, \rho_{0}=0.153 \mathrm{fm}^{-} 3$, $a_{v}=16.23 \mathrm{MeV}$. The FSU EoS uses the outer crust adopting the Baym, Pethick and Sutherland (BPS) model [23], whereas the DD2 EoS use the same Lagrangian for the low density part and allows a smooth and thermodynamically consistent transition to the high density part. A proper core-crust matching is very crucial to avoid uncertainties in the macroscopic properties of the stars as emphasised in Ref. [24]. Both the nuclear model EoSs are compatible with available astrophysical observational constraints. Firstly, they obey the maximum mass constraint from astrophysical observations [2527]. Also, they are consistent with the tidal deformability constraint $\Lambda_{1.4} \simeq 800$, inferred from the first analysis of GW170817 event (for NS of mass $M=1.4 M_{\odot}$ ) [14].

We consider additional strange components along with the nucleons only EoSs. The presence of any strange particle is expected to soften the EoS and lower the maximum mass of the NS, However, one can still manage to construct an EoS with strange matter and achieve a maximum mass within the observable limit. Here, we consider two sets of strange particles i.e. $\Lambda$ hyperons, and quarks in addition to the nucleons. To include the lightest baryon $\Lambda$ hyperons, an additional $\phi$-mesons are introduced for hyperonic interaction in addition to $\sigma, \omega$ and $\rho$ mesons of usual density dependent relativistic field theoretical model [28]. The SU(6) relations are exploited to determine the hyperon-vector meson couplings, and hypernuclei data to determine hyperon-scalar meson couplings. Finally, for the quark phase in the NS interior, we adopt the widely-used phenomenological MIT bag model [29]. The Bag constant $B^{1 / 4}$ is taken as $150 \mathrm{MeV}$.
This parameter can be adjusted to make the EoS consistent with the mass-radius observations [30]. The hadronic part is described within FSUGarnet model.

\section{Numerical model and results}

We now present our results for both the methods described in Sect. 2. We start with plotting the EoS, mentioned in the previous Section and the corresponding structure profile in GR.

In Fig. 1a, the pressure (p) vs energy density $(\rho)$ is plotted for the the nucleonic EoS HS(DD2) and FSUGarnet in solid lines. The DD2 EoS is named $\mathrm{BHB} \Lambda \phi$ as per the original nomenclature when $\Lambda \mathrm{s}$ are added to it [28]. On the other hand, FSUQ EoS has quarks on top of the nucleonic FSUGarnet EoS [20]. The strange BHB $\Lambda \phi$, and FSUQ EoSs are plotted in dashed and dotted lines respectively. Of the two nucleonic EoSs considered, HS(DD2) equation is visibly much stiffer than FSU. The addition of hyperons/quarks to nucleonic EoS has made them softer. Also, FSUQ is softer than $\mathrm{BHB} \Lambda \phi$.

In GR a static, spherically symmetric, non-rotating, hydrodynamic equilibrium configurations are obtained by solving the TOV equations (Eq. 2) with the appropriate boundary conditions for pressure and mass at the center and at the surface for each EoS, with the integration performed from the center to the surface of the NS. In Fig. 1b, the GR mass vs radius for $\mathrm{HS}(\mathrm{DD} 2)$, BHB $\Lambda \phi$, FSU, and FSUQ are plotted. The maximum masses for each of them are listed in Table 1 . The portion of the curves, where $\frac{d M}{d \rho_{c}}<0$ is gravitationally unstable. The black horizontal line represents the maximum mass constraint from the observation of $2 M_{\odot}$ and the grey band represents the error of the measurement. It can be seen that these four EoS satisfy the maximum mass constraint. The addition of hyperons to HS(DD2) EoS reduced the maximum mass from $2.42 M_{\odot}$ to $2.09 M_{\odot}$. The addition of quarks to FSU EoS reduced the maximum mass from $2.06 M_{\odot}$ to $2.00 M_{\odot}$. However, the radius corresponding to maximum mass of all these EoS are within 11.4-11.9 km, which is well within the NICER results.

In $f(R)$, we are studying the comparison of perturbative and non-perturbative approaches. In perturbative approach, we derive the TOV equations based on the assumption that the effects are just the higher order corrections to GR. The modified TOV equations thus obtained are solved numerically with boundary conditions for pressure and mass at the center and at the surface for each EoS. The Schwarzschild exterior solution is imposed, which is akin to freezing the scalar degree of freedom outside the star. In the non-perturbative approach, the interior and exterior equations are solved selfconsistently and non-perturbatively.

In Fig. 2a, the mass vs radius for HS(DD2) EoS is plotted both for perturbative approach (dashed lines) and non- 


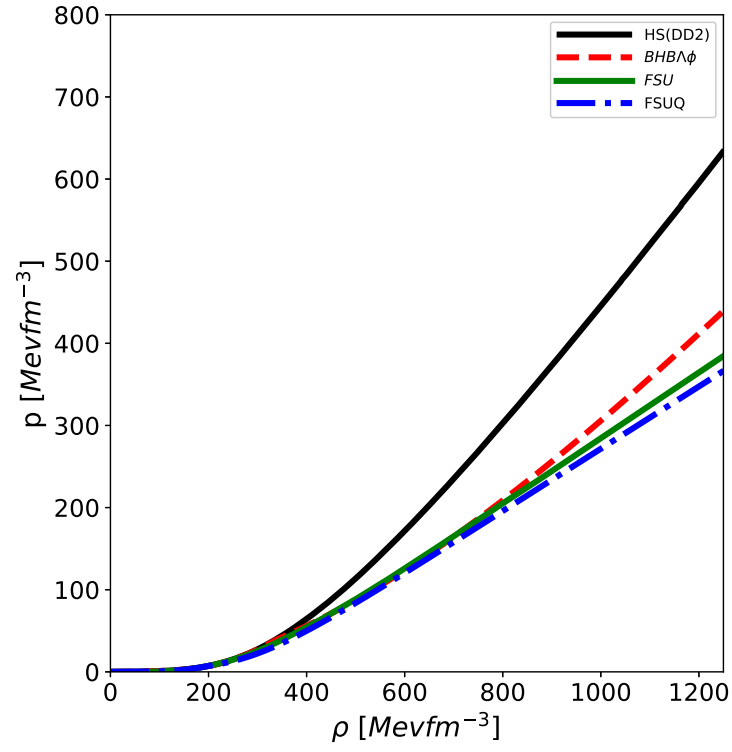

(a)

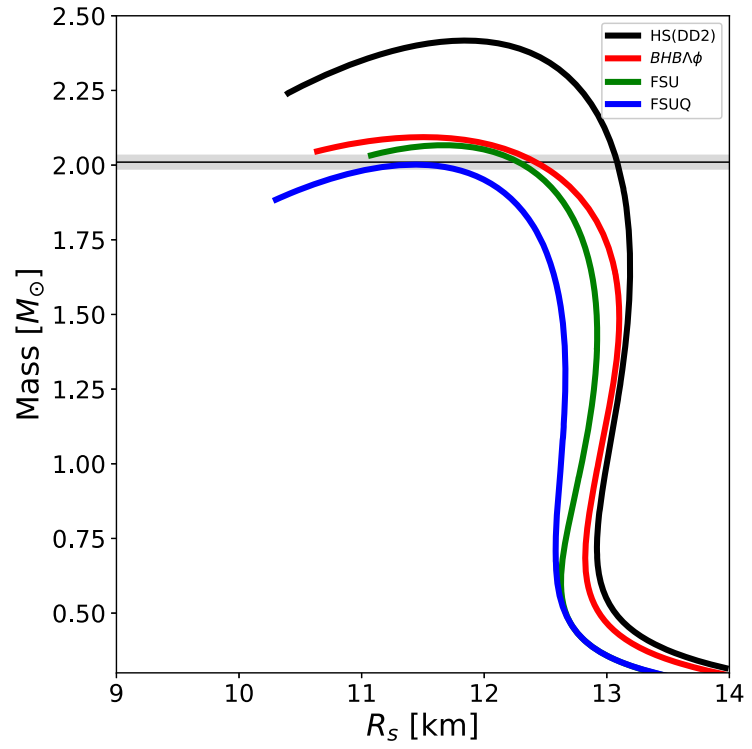

(b)

Fig. 1 a Pressure vs energy density curve of the EoS under study. b GR MR curves of EoS

Table 1 Maximum mass and corresponding radius of the NS for $a=0(\mathrm{GR}) ; a=50$ for non-perturbative (NP) $f(R)$ and $a=0.2$ for perturbative (P) $f(R)$

\begin{tabular}{|c|c|c|c|c|c|c|}
\hline \multirow[t]{2}{*}{ EoS } & \multicolumn{2}{|l|}{ GR } & \multicolumn{2}{|c|}{ NP f(R) } & \multicolumn{2}{|l|}{$P f(R)$} \\
\hline & $\begin{array}{l}M_{\max } \\
M_{\odot}\end{array}$ & $\begin{array}{l}\text { Radius } \\
\mathrm{km}\end{array}$ & $\begin{array}{l}M_{\max } \\
M_{\odot}\end{array}$ & $\begin{array}{l}\text { Radius } \\
\mathrm{km}\end{array}$ & $\begin{array}{l}M_{\max } \\
M_{\odot}\end{array}$ & $\begin{array}{l}\text { Radius } \\
\mathrm{km}\end{array}$ \\
\hline DD2 & 2.42 & 11.85 & 2.66 & 13.06 & 1.99 & 10.32 \\
\hline $\mathrm{BHB} \Lambda \phi$ & 2.09 & 11.51 & 2.34 & 12.53 & 1.99 & 9.91 \\
\hline FSU & 2.07 & 11.66 & 2.31 & 12.59 & 1.67 & 10.11 \\
\hline FSUQ & 2.00 & 11.44 & 2.24 & 12.41 & 1.61 & 9.91 \\
\hline
\end{tabular}

perturbative approach (dotted lines). The maximum mass in the perturbative approach decreases as the value of the free parameter $a$ increases. For values of $a$ greater than 0.2 , the maximum mass reduces below the observational limit. The maximum mass in the non-perturbative approach, however, increases with increase in the parameter $a$ with a marginal increase in radius. In this approach, we have considered values of $a$ up to 100 which is at the upper limit in sensitivity of the current gravitational wave detectors. In Fig. 2b, the mass vs radius for $\mathrm{BHB} \Lambda \phi \mathrm{EoS}$ is plotted both for perturbative approach (dashed lines) and non-perturbative approach (dotted lines). The maximum mass in the perturbative approach decreases as we increase the free parameter $a$, and reaches well below the observational limit even for a increase in $a$ of 0.1 . The maximum mass in the non-perturbative approach, however, increases with increase in the parameter $a$ and stays above the observational constraint with a marginal increase in radius.

In Fig. 3a, the mass vs radius for FSU EoS is plotted both for both perturbative (dashed lines) and non-perturbative (dotted lines) approaches. In the perturbative approach, we see that as we increase value of the free parameter $a$, the maximum mass decreases. For value of $a=0.1$, the maximum mass reduces well below the observational limit. In the non-perturbative approach, however, the maximum mass stays above the observational constraint for all values of $a$ up to 100 . In Fig. 3b, the mass vs radius for FSUQ EoS is plotted both for perturbative (dashed lines) and non-perturbative (dotted lines) approaches. The maximum mass in the perturbative approach falls well below the observational limit even for a increase in $a$ of 0.1 . The maximum mass in the nonperturbative approach, however, increases with increase in the parameter $a$ and stays above the observational constraint with a slight increase in radius of NS.

In Fig. 4a, the variation in compactness with respect to change in $a$ is plotted for the non-perturbative approach. We see that the for any given $a$, the compactness of the NS is correlated with the stiffness of the EoS, with the stiffest of the EoS, HS(DD2), having the highest compactness and the softest of the EoS, (FSUQ), having lowest compactness. The 


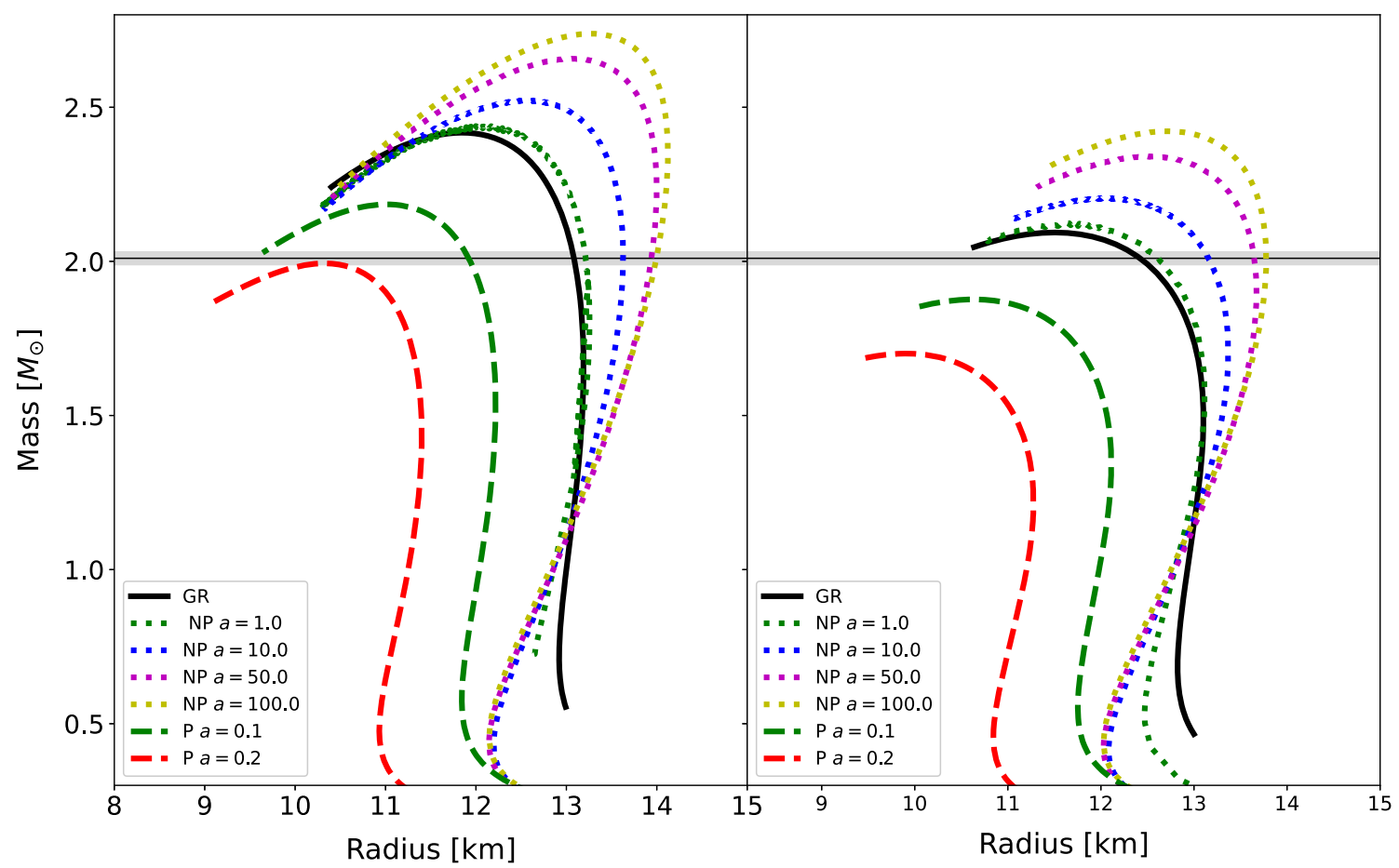

(a)

(b)

Fig. 2 a MR curves of EoS DD2 obtained using both perturbative (dashed lines) and non-perturbative (dotted lines) methods. b MR curves of EoS B $H B \Lambda \phi$ obtained using both perturbative (dashed lines) and non-perturbative (dotted lines) methods

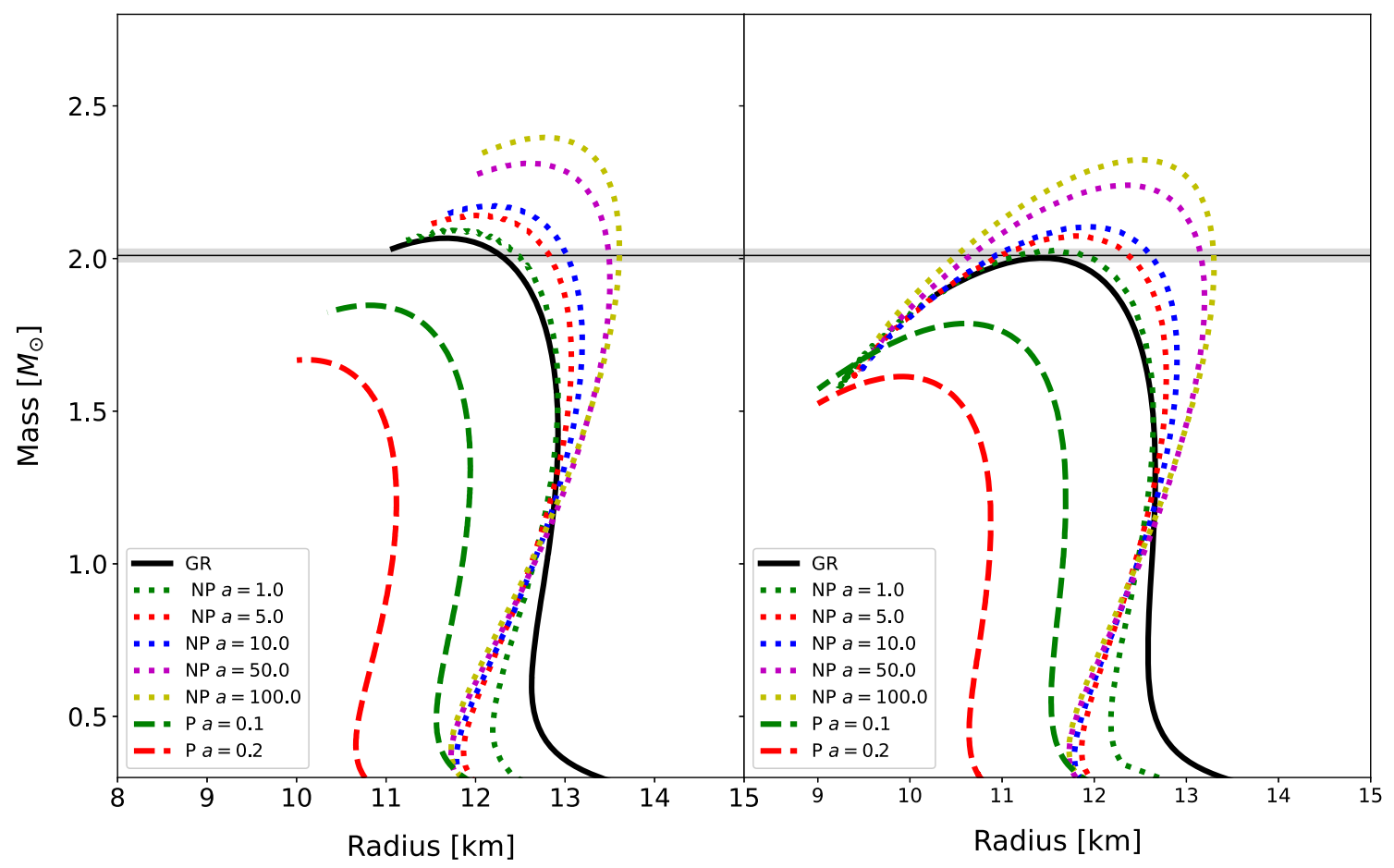

(a)

(b)

Fig. 3 a MR curves of EoS FSU obtained using both perturbative (dashed lines) and non-perturbative (dotted lines) methods. b MR curves of EoS FSUQ obtained using both perturbative (dashed lines) and non-perturbative (dotted lines) methods 


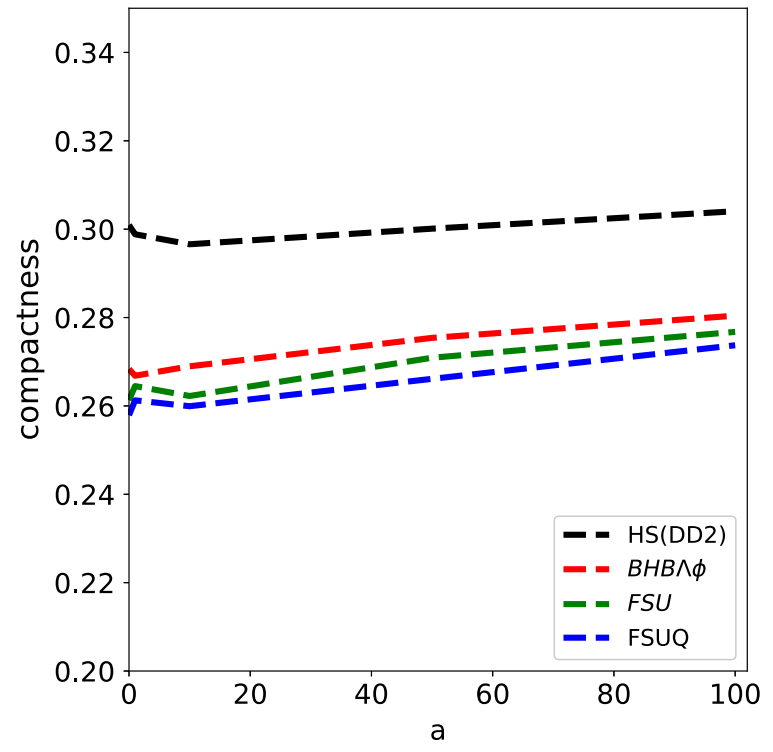

(a)

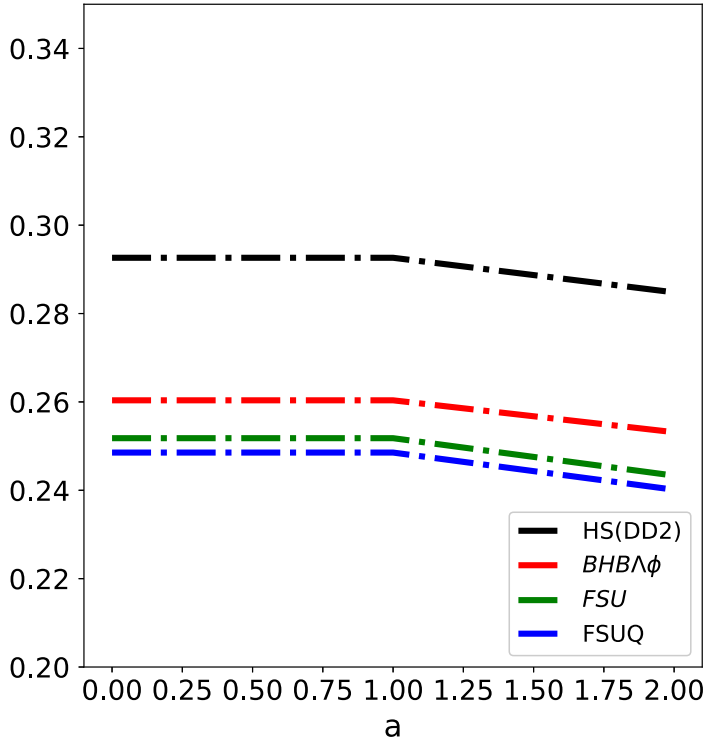

(b)

Fig. 4 a Compactness of NS for different EoS using non-perturbative method. b Compactness of NS for different EoS using perturbative method

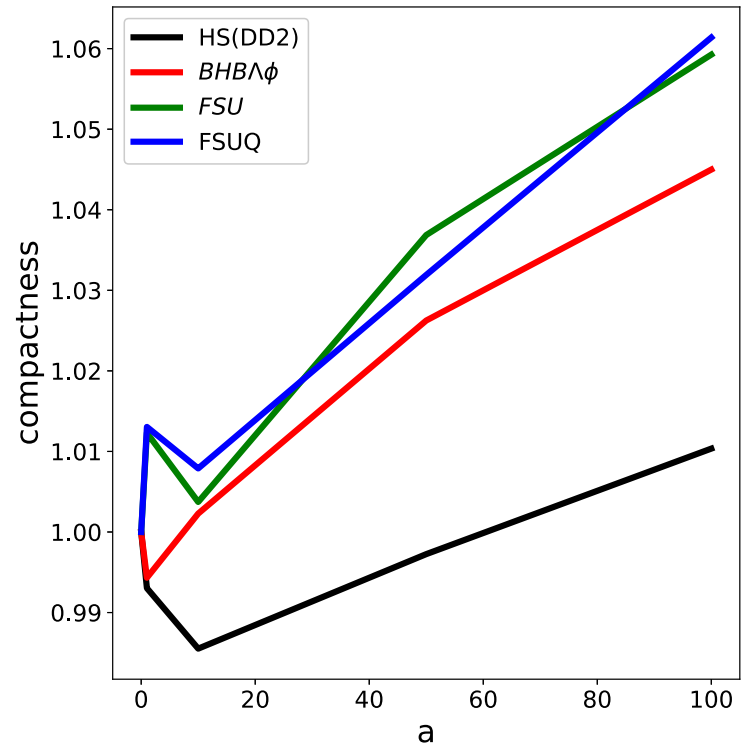

(a)

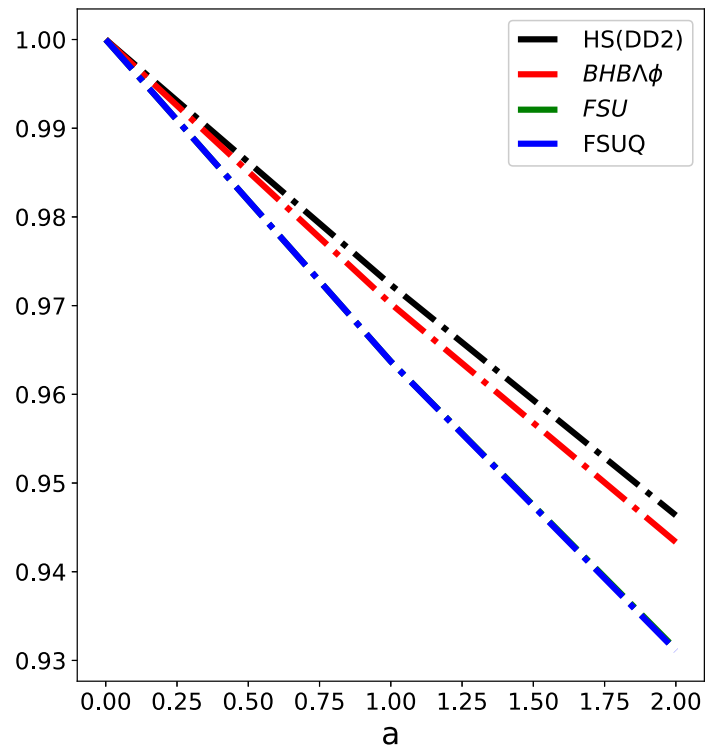

(b)

Fig. 5 a Compactness normalized with respect to the GR compactness for non-perturbative method. b Compactness normalized with respect to the GR compactness for perturbative method

addition of hyperons and quarks to the nucleonic EoS reduces the compactness of NS. Initially, as the value of $a$ increases, the compactness decreases and then increases steadily. In Fig. 4b, compactness vs $a$ is plotted for the perturbative approach. We see that the stiffness of the EoS is correlated with the compactness of the NS. However, the overall compactness of the NS is less than that of the non-perturbative approach and as the value of $a$ increases, the compactness decreases.
In Fig. 5a, the compactness of the NS obtained in $f(R)$ is normalized to the compactness of the NS obtained in GR. In non-perturbative approach, we see that for $a<10$ the compactness reduces and then increases steadily. It is interesting to note that softer EoS NS are more compact in $f(R)$ compared to GR. The addition of quarks/hyperons increases the compactness with respect to GR as $a$ increases. In the perturbative approach, the compactness decreases as $a$ increases. 


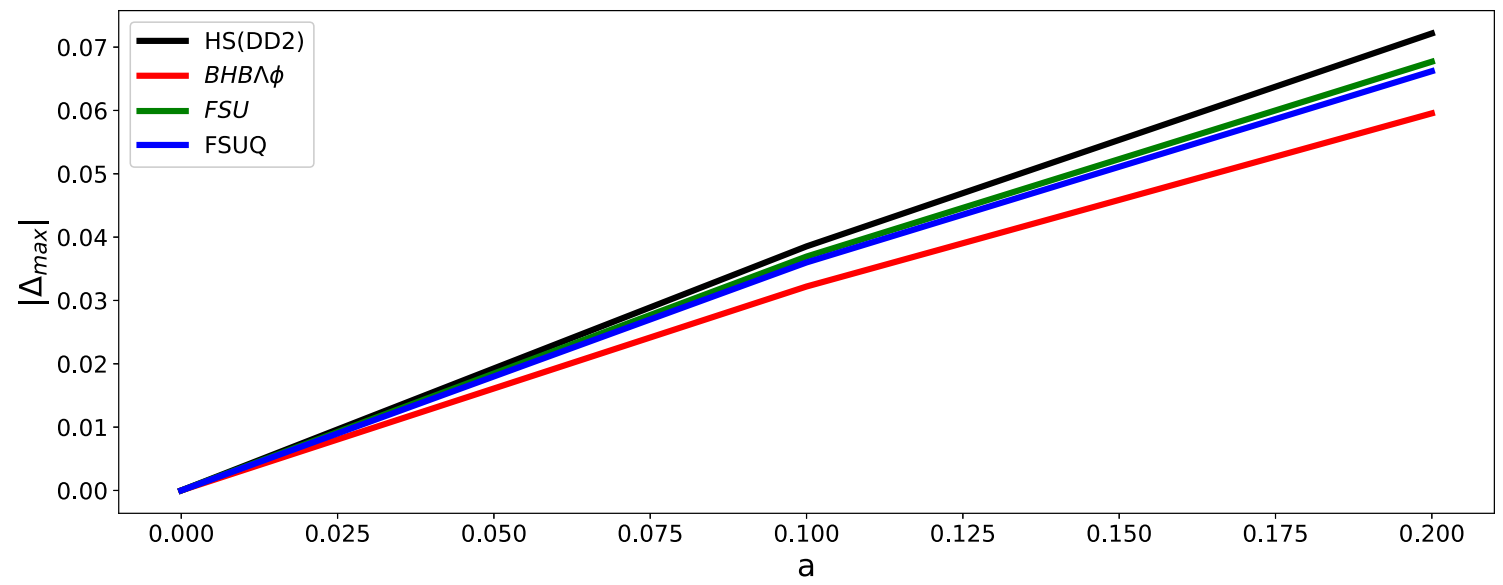

Fig. $6\left|\Delta_{\max }\right|$ as a function of $a$ for all EoSs in $f(R)$

Compactness of softer EoS is less than the compactness of stiffer EoS.

For all the cases considered here with respect to different values of $a$ in $f(R)$ model for the various EoS, the maximum allowed deviation from the GR prediction is constrained by the requirement that the solutions hold their perturbative validity. To be within the perturbative regime it is important that the first order corrections to the metric are small. This can be measured with

$|\Delta|=\left|\frac{A_{f(R)}(r)}{A_{G R}(r)}-1\right|$

where $A_{f(R)}(r)$ and $A_{G R}(r)$ are the $r r$ component of the metric defined for $f(R)$ and GR, respectively. This quantity is a function of radius of each star and depends on the EoS. It has a maximum value near the core of the star, where the density and the curvature is large. As we need the entire solution to be perturbatively close to the GR, we evaluate $\Delta$ at its maximum. The necessary condition for perturbative validity is that the maximum ratio $\left|\Delta_{\max }\right|<1$.

Figure 6 shows $\left|\Delta_{\max }\right|$ as a function of parameter $a$ for the EoS in $f(R)$ gravity model. This figure demonstrates that NS in the $f(R)$ gravity model can certainly be treated perturbatively as long as the magnitude of $a$ is in the range $0<a<0.2$.

\section{Conclusion}

Neutron Stars are the densest stellar object where matter is in extremely high gravitational field regimes, hence are important tools to test alternative theories of gravity. The study of NS is important in relativistic astrophysics as they are key to understand the final stages of stellar evolution. NS in the background of modified theories of gravity undergoes correction in the Tolman-Oppenheimer-Volkoff (TOV) equations of stellar hydrostatic equilibrium. This change has a huge impact on the observational properties of the NS. The uncertainty in the equation of state of matter at immensely high-density core of NS along with the new parameters of the modified theory of gravity under consideration provides a completely new phenomenology for the typical predictions of stable stellar structure models, such as maximum masses and mass-radius relations. One can then tally these with the astrophysical observations to constrain the modified gravity model for its viability.

In this paper, we analyze the NS solutions with realistic EoS following two approaches namely, perturbative and nonperturbative $f(R)$ gravity. For this, we choose EoSs, that are generated in the framework of relativistic mean field models. The NS core under these EOSs, contains the strange particles in the form of $\Lambda$ hyperons and quarks as well as nucleons and leptons. With these EOSs, the mass-radius profiles for static NSs, calculated in GR by solving the TOV equations lie well within the observational limits.

Obtaining the TOV equations from the fourth order field equation is difficult. Commonly two methods are used to solve these equation in case of $f(R)$ gravity, the pertubative method, where $f(R)$ theories are considered as a perturbation to GR and the non pertubative method. We use both these methods in $R^{2}$ gravity and try to figure out which method is more suited to investigate the strong field regime. Our numerical integration using nuclear EoSs (DD2, FSU Garnet) and strange $\operatorname{EoSs}(B H B \Lambda \phi, F S U Q)$, constraints the extra degree of freedom parameter $a$ that arise in in $f(R)=R+a R^{2}$ gravity. It is to be noted that for $a=0$ the maximum mass tends to the corresponding value in GR for the EoS.

For the case of non-perturbative method, a wide range of parameter $a$ is considered. We notice that, for all $a$ more massive the star, more is its compactness. Once the maximum possible mass is attained, the star configuration becomes unstable. The maximum mass of NS is always greater than 
in case of GR and is larger for large values of $a$. The radius of the NS too increases marginally with increase in $a$. This trend is same for both nuclear and strange EoSs, however, the strange EoSs NS are more compact in $f(R)$ compared to GR. Although, for nonzero values of $a$ the deviation from GR can be large, they are still admissible, as they satisfy the observational bound of $2 M_{\odot}$ limit. This is why the present observations of the NS masses and radii can not alone put constraints on the value of the parameter $a$. We can therefore conclude that $f(R)$ theory of gravity can brings about remarkable change in the properties of NS. Also, $f(R)$ can admit the EoSs which otherwise do not satisfy the observational limit of $2 M_{\odot}$ in GR.

The perturbative approach in $f(R)=R+a R^{2}$ can be used only when the range of $a$ is $0<a<0.2$. In this limit, except for EoS DD2 none other EoSs considered satisfy the observational limit of maximum mass $\left(2 M_{\odot}\right)$. This approach therefore doesn't seem to work in the strong field limit and does not satisfy the astrophysical observations of NS. The main reason behind it is we do not consider the nonlinear terms which might appear in the strong field regime with repercussions on the structure and the properties of the Neutron Stars. Many variable modified gravity models have been studied in literature using the perturbative approach [31-33], where negative values of the free parameter is considered to fit the observations. However illustrative studies in Ref. $[34,35]$ show that a viable gravity with negative free parameter is not feasible due to presence of ghost and tachyonic instabilities.

Acknowledgements Authors thank Tuhin Malik for the useful discussions. AA is thankful to UGC for providing financial support under the scheme Dr. D.S. Kothari postdoctoral fellowship.

Data Availability Statement This manuscript has no associated data or the data will not be deposited. [Authors' comment: There are no associated data available.]

Open Access This article is licensed under a Creative Commons Attribution 4.0 International License, which permits use, sharing, adaptation, distribution and reproduction in any medium or format, as long as you give appropriate credit to the original author(s) and the source, provide a link to the Creative Commons licence, and indicate if changes were made. The images or other third party material in this article are included in the article's Creative Commons licence, unless indicated otherwise in a credit line to the material. If material is not included in the article's Creative Commons licence and your intended use is not permitted by statutory regulation or exceeds the permitted use, you will need to obtain permission directly from the copyright holder. To view a copy of this licence, visit http://creativecomm ons.org/licenses/by/4.0/.

Funded by SCOAP ${ }^{3}$.

\section{References}

1. A. Einstein, Ann. Phys. 49, 769-822 (1916)

2. S. Perlmutter et al., Supernova Cosmology Project Collaboration, Astrophys. J. 517, 565 (1999)

3. A.G. Riess et al., Supernova Search Team Collaboration, Astron. J. 116, 1009 (1998)

4. D.N. Spergel et al., WMAP Collaboration, Astrophys. J. Suppl. 170, 377 (2007)

5. U. Seljak, A. Makarov, P. McDonald, S.F. Anderson, N.A. Bahcall, J. Brinkmann et al., SDSS Collaboration, Phys. Rev. D 71, 103515 (2005)

6. S. Das, E.C. Vagenas, Universality of quantum gravity corrections. Phys. Rev. Lett. 101, 221301 (2008)

7. T. Clifton, P.G. Ferreira, A. Padilla, C. Skordis, Modified gravity and cosmology. Phys. Rep. 513 (2012)

8. T.P. Sotiriou, V. Faraoni, $\mathrm{f}(\mathrm{R})$ theories of gravity. Rev. Mod. Phys. 82, 451 (2010)

9. A.V. Astashenok, AIP Conf. Proc. 1606, 99 (2014)

10. S. Arapoglu, C. Deliduman, K. Yavuz Eksi, J. Cosmol. Astropart. Phys. 7, 20 (2012)

11. K Nobleson, A. Ali, S. Banik, arXiv:2010.15406

12. S.S. Yazadjiev, D.D. Doneva, K.D. Kokkotas, K.V. Staykov, J. Cosmol. Astropart. Phys. 06, 003 (2014) Staykov., Journal of Cosmology and Astroparticle Physics, no. 06 (2014): 003

13. K Nobleson, T. Mallik, S. Banik, arXiv:2105.07813

14. B.P. Abbott et al., Phys. Rev. Lett. 119, 161101 (2017)

15. B.P. Abbott et al., The LIGO Scientific Collaboration and the Virgo Collaboration, Phys. Rev. Lett 121(16), 161101 (2018)

16. G. Raaijmakers et al., Astrophys. J. 887(1), 22 (2019)

17. M.C. Miller et al., Astrophys. J. 887(1), 24 (2019)

18. M. Hempel, J. Schaffner-Bielich, Nucl. Phys. A 837, 210 (2010)

19. P. Char, S. Banik, Phys. Rev. C 90, 015801 (2014)

20. Wei-Chia Chen, J. Piekarewicz, Phys. Lett. B 748, 284 (2015)

21. J.D. Walecka, Ann. Phys. 83, 491 (1974)

22. S. Typel, G. Röpke, T. Klähn, D. Blaschke, H.H. Wolter, Phys. Rev. C 81, 015803 (2010)

23. G. Baym, C. Pethick, P. Sutherland, Astrophys. J. 170, 299 (1971)

24. M. Fortin, C. Providência, Ad.R. Raduta, F. Gulminelli, J.L. Zdunik, P. Haensel, M. Bejger, Phys. Rev. C 94, 035804 (2016)

25. E. Fonseca et al., Astrophys. J. 832(2), 167 (2016)

26. J. Antoniadis et al., Science 340, 448 (2013)

27. P.B. Demorest, T. Pennucci, S.M. Ransom, M.S.E. Roberts, J.W.T. Hessels, Nature 467, 1081 (2010)

28. S. Banik, M. Hempel, D. Bandyopadhyay, Astrophys. J. Suppl. 214, 22 (2014)

29. A. Chodos, R.L. Jaffe, K. Johnson, C.B. Thorne, Phys. Rev. D 10, 2599 (1974)

30. R. Nandi, P. Char, S. Pal, Phys. Rev. C 99, 052802(R) (2019)

31. Artyom V. Astashenok et al., JCAP 01, 001 (2015)

32. Artyom V. Astashenok et al., JCAP 12, 040 (2013)

33. V. Artyom, A.S. Capozziello, S.D. Odintsov, Phys. Rev. D 89, 103509 (2014)

34. Artyom V. Astashenok et al., Class. Quantum Gravity 34, 205008 (2017)

35. A. De Felice, S. Tsujikawa, Living Rev. Relativ. 13, 3 (2010) 